\title{
ANAESTHESIA FOR CARDIAC PATIENTS HAVING NON-CARDIAC OPERATIONS*
}

\author{
J. EARL WYNANDS
}

ANAESTHESIA for patients with heart disease undergoing cardiac and non-cardiac surgery has been the subject of previous reviews. ${ }^{1-4}$ The purpose of this presentation is to emphasize factors which we believe are important in reducing morbidity and mortality in patients with heart disease undergoing non-cardiac surgery.

It is enlightening and, at the same time, disturbing that the high reinfarction rate reported by Topkins and Artusio in $1964^{5}$ on studies done from 1959 to 1962 was only slightly improved by $1967 / 68$, as reported by Tarhan, et al. ${ }^{6}$ The latter group reported that patients who were operated on within three months of infarction had a 37 per cent reinfarction rate. This rate decreased to 16 per cent in patients at three to six months after infarction, and remained at four to five per cent when infarction had occurred more than six months previously. They also identified that operations on the thorax and upper abdomen had three times as many reinfarctions as operations at other sites. A significantly higher number of myocardial infarctions occurred during the third postoperative day. A follow-up study by Steen, et al in $1974 / 75^{7}$ showed no significant change in reinfarction rates. As with previous studies, there was no correlation between reinfarction and anaesthetic techniques or agents. Reinfarction was significantly higher in patients whose operations lasted more than five hours. Co-existence of diabetes, angina and hypertension increased the reinfarction risk threefold. Hypotension during operation defined as a 30 per cent fall from control, was associated with a five-fold increase in reinfarction rate. These results were surprising because, by that time, the concept of the myocardial oxygen balance (Figure 1) had been established. ${ }^{\perp}$ Anaesthetists could now not only "avoid hypoxia and hypotension" but could alter myocardial oxygen demands in patients with heart disease. We were

J. Earl Wynands, M.D., C.M., F.R.C.P.CC), Associate Professor, Departments of Anaesthesia \& Surgery, McGill University; Senior Anaesthetist, Royal Victoria Hospital; Montreal, Quebec.

*Presented in a Panel Discussion of Cardiac Anaesthesia and Surgery at the Annual Meeting, Canadian Anaesthetists' Society, Halifax, N.S., June 1981. then in a position to alter preload, heart rate, systemic vascular resistance, and the contractile state of the heart.

We have for some time held the opinion that, although patients may sustain a myocardial infarction in the operating room, in general they are carefully monitored and managed in this period and they may be at greater risk in the post-operative phase. When it has been established that their recovery room condition is stable, they are discharged to ward care. This is in contrast to patients with heart disease undergoing open heart surgery. These patients for the most part have their cardiac disability improved if not repaired but then are carefully observed and monitored for 24 to 48 hours in the recovery room. Frequently unexpected therapeutic interventions are initiated late in the recovery room stay of these cardiac surgery patients to prevent or treat complications. It is not surprising then that Tarhan, et al. ${ }^{6}$ found, in non-cardiac surgery patients, a significant increase in reinfarction rate on the third postoperative day, a time when these patients are most likely to be back on the ward with limited capabilities for observation.

The level to which perioperative myocardial reinfarction can be reduced has yet to be determined and will be based on the identification of the high risk patient whose cardiac performance must be closely monitored and appropriate interventions made throughout the perioperative period. This concept is supported by a recent report by Rao and El-Etr ${ }^{8}$ who studied 97 patients with a history of previous myocardial infarction undergoing general surgery from July

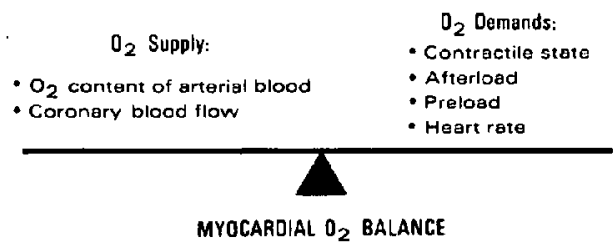

FIGURE 1 Factors in critical balance between oxygen supply and demand of the myocardium. (Reprinted with the permission of the Canadian Journal of Surgery, Volume 21, No. 6, 1978.)

Can. Anaesth. Soc. J., vol. 29, no. 4, July 1982 
1977 to June 1980 . They reported reinfarction rates in patients who had infarction within three months to be 7.8 per cent and a postoperative mortality of 5.3 per cent. In patients who had infarction within three to six months reinfarction rate was 3.4 per cent and there was no mortality. This is in marked contrast to previous studies. They concluded that close haemodynamic monitoring with immediate and appropriate therapy to control various haemodynamic aberrations contributed to the lower reinfarction rate in this group of high risk patients.

These interventions presume a knowledge of the pathophysiology of heart disease and, in this instance, we will be particularly concerned with ischaemic heart disease.

\section{Pathophysiology of Ischaemic Heart DISEASE}

The normal heart may be conveniently thought of as four chambers in series (Figure 2). For all practical purposes, all chambers may be considered to have equal volumes. The muscle mass of the left ventricle is the largest of the four chambers, as it is a pressure pump compared to the right ventricle, which has a smaller muscle mass and is a volume pump. Both the right and left sides of the heart work against a resistance, that on the left side (systemic vascular resistance) being 10 times higher than the pulmonary vascular resistance faced by the right ventricle.

Unlike other organs, the heart serves as the source of its own blood supply through the

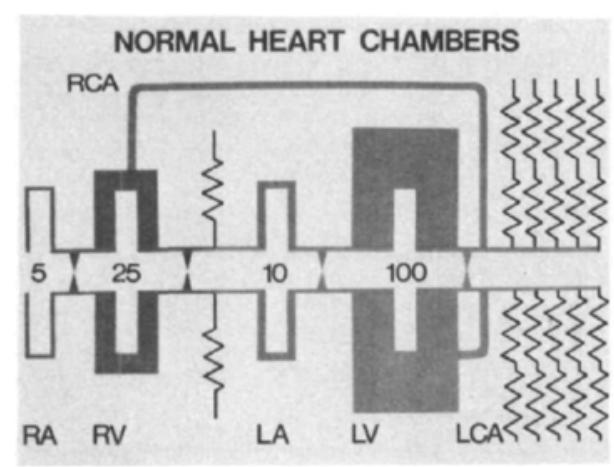

FIGURE 2 RA - right atrium; RV - right ventricle; LA - left atrium; LV - left ventricle; LCA - left coronary artery; RCA - right coronary artery. The four chambers are of equal volume but vary in muscle mass. The right and left sides of the heart have different filling pressures, generate different pressures and work against marked differences in resistance. coronary vessels. There are differences in blood flow to the right and left sides of the heart. Blood flow to the left side occurs only during diastole, whereas that to the right ventricle occurs during both systole and diastole. The reason for this is related to the differences between the coronary artery diastolic perfusion pressure and the combined pressure of the intrinsic mass of the left ventricle as compared to the right ventricle and the end diastolic pressures.

Effective cardiac function depends not only on an adequate coronary blood flow and nutritional content, but also upon effective function of the valves which separate chambers on each side from one another and the resistance against which each side must work.

Finally, the ultimate function of the heart is to supply itself and all the tissues of the body with an adequate blood flow. This work of the heart involves pressure and volume; 75 per cent of the oxygen expenditure of the heart is related to internal work (isometric contraction), the remaining 25 per cent to external work (isovolumic contraction) and 95 per cent of this external work is overcoming peripheral vascular resistance. The heart, therefore, may fail to work for a number of reasons which include loss of muscle mass, myocardial depression, valvular dysfunction, increased peripheral vascular resistance, inadequate chamber filling and coronary blood flow insufficient to meet requirements of the myocardium. These over-simplified aetiological factors may lead to left, right or biventricular failure.

A schematic representation of left ventricular failure is shown in Figure 3. There is, for one reason or another, left ventricular dysfunction which results in a decrease in left ventricular cardiac output. This decrease in output will lead to a decrease in blood pressure which, in turn, will reflexly increase heart rate and systemic vascular resistance and stimulate the renin angiotensin system. ${ }^{9}$ The increase in heart rate decreases the myocardial diastolic perfusion time upon which the left ventricle depends for its blood supply. The end-diastolic pressure of the left ventricle (LVEDP) will rise and this will be reflected by an increase in the left atrial pressure. The increase in heart rate, decrease in aortic diastolic pressure and increase in LVEDP will especially impair endocardial blood flow and aggravate myocardial depression. Over a protracted period of time, in particular if there is mitral valvular insufficiency, increased left atrial pressure will lead to an increase in pulmonary 


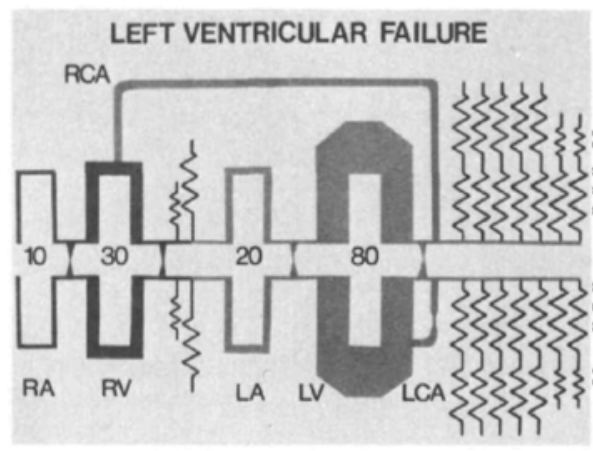

FIGURE 3 RA - right atrium; RV - right ventricle; LA - left atrium; LV - left ventricle; LCA - left coronary artery; RCA - right coronary artery. There is impaired left ventricular function. When severe, systemic pressure is reduced and there are elevations in SVR and left-sided end-diastolic volumes and pressures, the right heart will work against a higher resistance (pulmonary hypertension) which may result in an increase in its filling pressure.

vascular resistance. A prolonged increase in pulmonary vascular resistance will impose a strain on the right ventricle and may lead to an increase in its end-diastolic volume and pressure which will be reflected by an increase in the right atrial pressure (CVP). It must be emphasized, however, that when the left ventricle fails, for whatever reason, there may be adequate coronary blood flow to the right ventricle to allow it to perform for a short period of time in a relatively normal manner. Thus the right ventricle can, for minutes if not hours, pump more blood to the left ventricle than the latter can accommodate. This is the concept of ventricular mismatch ${ }^{10}$ which will be detected by a decreasing systemic blood pressure, a fall in cardiac output, an increase in left atrial pressure, a significant increase in pulmonary artery pressure with a slightly high or normal central venous pressure.

When right ventricular failure occurs, for whatever reason, filling pressures on the left side of the heart will be low normal and the central venous pressure, which reflects the right atrial pressure, will be high. The pulmonary artery pressure, depending on the aetiology of the right ventricular failure, may be high or low. Pulmonary vascular resistance will probably be reflexly increased because of a reduction in cardiac output when there is right ventricular failure. This reduction in cardiac output will also increase systemic vascular resistance and heart rate. There will be an increase in the end-dia- stolic pressure of the right ventricle which, if sufficiently high and associated with a decrease in coronary artery perfusion pressure, may result in coronary blood flow occurring only during diastole, as is the situation on the left side of the heart (Figure 4).

The most severe form of heart failure is represented in Figure 5 and termed biventricular failure. There is marked impairment of left and right ventricular function and a decrease in cardiac output. Intense stimulation of the sympathetic nervous system will lead to marked increases in both the systemic vascular and pulmonary vascular resistances. There will be an increase in the heart rate and increases in end-diastolic volumes and pressures on both sides of the heart. Blood flow to both the left and

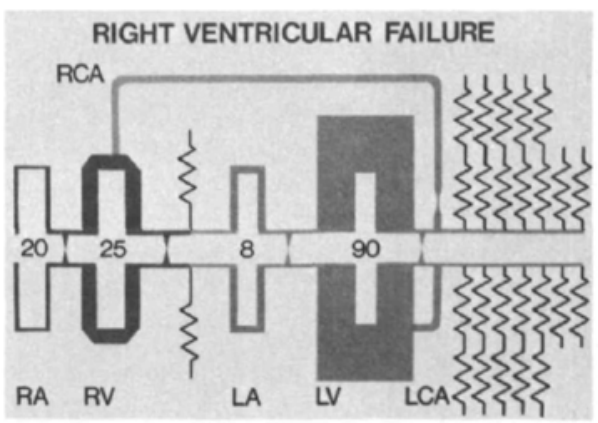

FIGURE 4 RA - right atrium; RV - right ventricle; LA - left atrium; LV - left ventricle; LCA - left coronary artery; RCA - right coronary artery. The right side can fail, decreasing the preload to the left. Right sided filling pressure will be high while that on the left is low. SVR will be elevated.

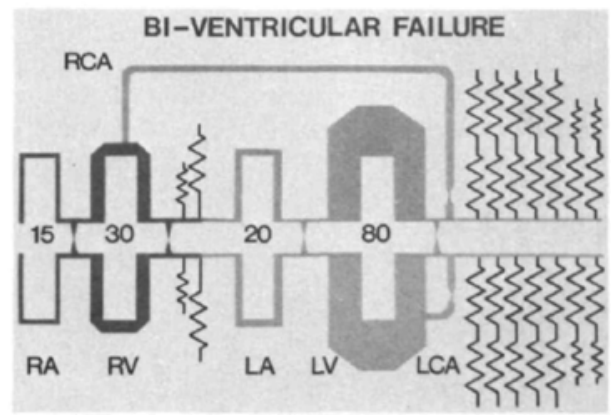

FIGURE 5 RA - right atrium; RV -right ventricle; LA - left atrium; LV - left ventricle; LCA - left coronary artery; RCA - right coronary artery. There is marked dysfunction on both sides of the heart with high filling pressures and high resistance. 
the right ventricles may occur only during diastole and, because of the increase in the end diastolic pressures and the reduction in the coronary artery diastolic perfusion pressure, there may be a marked impairment of blood flow to the sub-endocardium of both the left and right ventricles.

Factors which regulate the balance between the oxygen supply and the demands of the myocardium are represented in Figure 1. Maintenance of this critical oxygen balance is obviously of extreme importance in all patients with heart disease undergoing surgery.

The maintenance of cardiovascular system homeostasis ${ }^{11}$ may require interventions which will affect heart rate, the contractile state of the myocardium, peripheral vascular resistance and be associated with interventions that will alter the filling (preload) of the heart. Finally, it must be emphasized that the heart really does function as four chambers in sequence. They are divided into right and left sides, the effective function of which may vary and lead to ventricular mismatch which can only be diagnosed by appropriate monitoring.

\section{Perioperative Management}

\section{Preoperative Assessment}

The purpose of the preoperative assessment is to determine the patient's myocardial reserve. Factors which, if present, will indicate reduced reserve are a history of heart failure, angina particularly if it is changing or unstable, myocardial infarction, arrhythmias, electrocardiographic signs of ischaemia, diabetes, hypertension, obesity, and increasing age. Many of these factors have been taken into account by Gold$\operatorname{man}^{12}$ who has constructed a risk profile which is useful in predicting surgical outcome in the patient with cardiac disease. Cardiac catheterization studies showing occluded or markedly narrowed coronary arteries, increased filling volumes and pressures of the heart, areas of abnormal wall motion and an ejection fraction below normal indicate impaired reserve. This information is important to the anaesthetist because the patient with low myocardial reserve, as typified by patients with severe valvular disease or loss of muscle following coronary occlusion, may have reduced tolerance to anaesthesia, whereas patients with good myocardial reserve in spite of impaired coronary blood flow may have increased anaesthetic requirements and, indeed, may benefit from myocardial de- pression. ${ }^{13}$ The aim throughout the perioperative period should be to optimize cardiovascular system function, which becomes more important as the severity of heart disease increases. Effective treatment will require careful monitoring of cardiac haemodynamics. In general, flow work is desirable and resistance work above that which is required to perfuse vital organs adequately may be wasted. A slow heart rate is also desirable, not only because it reduces the oxygen requirements of the heart, but also because it favours myocardial diastolic perfusion time. The anaesthetist has available to him numerous pharmocological agents and mechanical devices which can be used to support the failing cardiovascular system. These include alpha and beta agonists, beta blockers, vasodilators, inotropic drugs such as calcium and digitalis, calcium antagonists, repolarizing solutions (glucoseinsulin-potassium), pacemakers and the intraaortic balloon counter pulsation device. ${ }^{11}$

\section{Monitoring}

Electrocardiogram: There is only time in this presentation to make short comments on the electrocardiogram (ECG), filling pressures, and rate pressure product as signs of myocardial dysfunction. Angina is the most valuable sign of myocardial ischaemia. This sign is lost to the anaesthetist in patients under anaesthesia and in the postoperative period where pain sensation may be altered by analgesic agents. The ECG thus serves as the only method of detecting myocardial ischaemia in the anaesthetized patient. It is generally agreed that lead V5 or CM5 of the ECG is most sensitive in detecting myocardial ischaemia and lead II is most appropriate for identification of supraventricular arrhythmias. It is only by critical appraisal of the ST segments of the ECG that myocardial ischaemia may be identified. Horizontal depression or downward sloping of the ST segment as well as ST elevation are signs of myocardial ischaemia. ${ }^{14}$ Too frequently, it would appear, the ECG is used only as a monitor of heart rate or, at best, to detect arrhythmias.

\section{Central Venous Pressure and Pulmonary Capillary Wedge Pressure}

The question always arises as to whether the central venous pressure (CVP) and/or the pulmonary capillary wedge pressure (PCWP) should be monitored in patients with heart disease. The answer to this question should be 
clear from the section on pathophysiology. The CVP reflects right ventricular loading or right ventricular end-diastolic pressure (RVEDP) whereas the PCWP relates to LVEDP. Although the CVP may bear a relationship to the LVEDP in patients with coronary artery disease who have a normal ejection fraction, normal heart wall motion and normal lungs, this relationship cannot be relied upon. ${ }^{16}$ Therefore, it is essential to know the LVEDP in patients with moderate to severe left heart dysfunction if treatment is to be appropriate. Furthermore, myocardial depression from whatever cause may lead to an increase in LVEDP which may interfere with endocardial blood flow. It is important to diagnose this event early in patients with a critical myocardial oxygen balance and this can only be accomplished with the use of a pulmonary artery catheter. It should be emphasized that, although an increase in the LVEDP may be subsequent to the inability of the heart to work against a high peripheral vascular resistance, a sudden increase in LVEDP may, however, be the first sign of coronary insufficiency producing myocardial depression. Reason would dictate that a pulmonary artery catheter should be used in all patients with moderate to severe myocardial dysfunction who are undergoing major surgical procedures such as vascular surgery, thoracic surgery, and operations in which large fluid volume shifts may be expected as in bowel obstruction. A CVP catheter should be used in all patients in whom a pulmonary artery catheter is not employed unless the extent of heart disease is minimal or the surgery brief and of small extent. The CVP will not only provide filling pressures of the right side of the heart which may correlate with the LVEDP, but will also serve as a central line for the rapid administration of any drugs which may be urgently required. There will always be patients in whom the decision to introduce a pulmonary artery catheter will not be clear cut and, in this situation, it is probably best to go along with the CVP but be prepared to introduce the former if information from a pulmonary artery catheter is deemed necessary.

\section{Rate Pressure Product (RPP)}

The product of the systolic blood pressure and the heart rate has been referred to as the rate pressure product (RPP) and accepted as an index of myocardial oxygen requirements. ${ }^{17,18} \mathrm{~Pa}$ tients who have ischaemic heart disease will have an RPP at which they will consistently exhibit signs or symptoms of myocardial is- chaemia (Figure 6). The question arises whether a RPP which is known to produce signs of myocardial ischaemia in the awake situation can be used as a predictor of ischaemia under anaesthesia. This question has not been satisfactorily answered but a reasonable approach would seem to be that if a RPP has been determined pre-operatively to be unsafe for an individual, the same should apply under anaesthesia. On the other hand, if it has been determined that a patient develops myocardial ischaemia at a RPP of $12,000,{ }^{14}$ which is a typical figure for patients with moderate to severe angina, one should not consider the patient under anaesthesia to be in a satisfactory condition if the RPP is 9,000 , if that figure is produced by systolic blood pressure of 60 torr and a heart rate of $160 \mathrm{bpm}$. If the numbers were reversed, then indeed the patient would probably not have myocardial ischaemia. Therefore, the heart rate is the more important component of the RPP. One should probably aim to maintain the heart rate between 60 and $70 \mathrm{bpm}$ and with this heart rate not to exceed a RPP which pre-operatively has been associated with signs or symptoms of myocardial ischaemia, or to accept a RPP which pre-operatively has been safe for the individual.

\section{Anaesthesia}

The choice of anaesthetic agent or technique is not as important as the ability of the anaesthetist to handle the agent or technique and his appreciation of the course to be followed or goals to be achieved if myocardial ischaemia and/or myo-

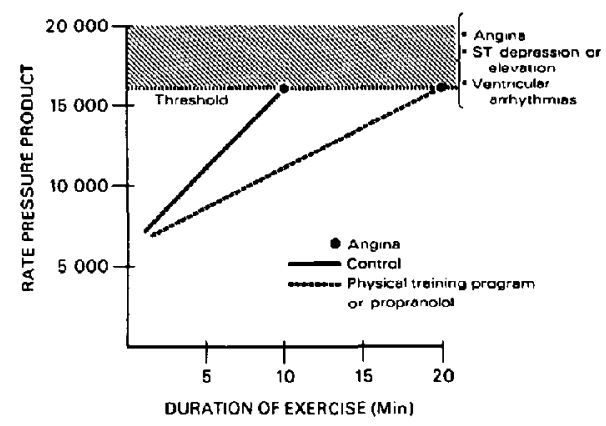

FIGURE 6 Rate pressure product (RPP), obtained by multiplying systolic blood pressure by heart rate. The RPP correlates well with myocardial oxygen consumption and patients with ischaemic heart disease will have a constant RPP at which signs or symptoms occur. (Reprinted with permission of the Canadian Joumal of Surgery, Volume 21, No. 6, 1978.) 
cardial dysfunction are to be avoided. There are operations and patients in whom regional anaesthesia has clear advantages over general anaesthesia. Operations on the extremities and perineum are particularly suited to regional anaesthesia, provided there is patient's acceptance. There is little point in doing an axillary block for an operation on an arm because this anaesthetic technique will cause little disturbance of haemodynamics if the patient objects to the procedure and it is anticipated that this apprehension cannot be allayed by explanation and the use of mild sedation. When the decision is made to manage patients with a general anaesthetic the warnings of our cardiology colleagues from the 50's and early 60 's should be adhered to; hypotension and hypoxia should be avoided. However, in the course of the ensuing 20 years we have come to learn that patients with ischaemic heart disease do better when their heart rate is about $60 \mathrm{bpm}$, not only because this reduces the oxygen requirements of the heart, but also because it allows for longer diastolic perfusion time of the left ventricle. In the last decade great emphasis has been placed on avoiding an increase of the oxygen requirements of the myocardium. ${ }^{19}$ Thus excessive increases in afterload or preload are to be avoided, as well as situations which may unnecessarily stimulate the contractile state of the myocardium. There can be no doubt that the patient who suddenly becomes tachycardic and hypertensive may develop alarming signs of ischaemia on the ECG. However the point must be made that, in avoiding hypertension, we should not unduly depress the cardiovascular system and produce hypotension that has been shown to be associated with reinfarction. Rather we should aim to maintain the patient's blood pressure within about 15 per cent of his ward pressure. Patients who are hypertensive should have it controlled by medical therapy before undergoing elective surgery ${ }^{20}$ and it is now generally accepted that patients who require propranolol for control of angina should have this drug continued up to the time of the operation. ${ }^{21}$ We believe that patients who have signs of ischaemic heart disease should be placed on propranolol therapy before anaesthesia and surgery unless they have contra-indications to it. Controlled myocardial depression may be deemed advisable 22 on the basis of information obtained from ongoing monitoring and this can be produced with any of the inhalation agents currently used, halothane being probably the most potent. When myocardial depression is to be avoided it has been our experience that it can be best accomplished using an oxygen and high dose fentanyl technique. ${ }^{23}$ It must be emphasized that haemodynamic stability is the central component of good anaesthesia in patients with heart disease. It is the fact that haemodynamic stability is attained rather than the manner in which it is attained that is important during anaesthesia and surgery.

The same aggressive attention to haemodynamic and respiratory function ${ }^{24}$ must be extended into the postoperative period if morbidity and mortality are to be reduced below the figures that have been recorded to the very recent past. This may create a logistic problem in terms of recovery room accommodation, where patients who are considered to be at high risk have to be kept for 24 to 48 hours after operation. Patients should not be discharged from Intensive Care until they are haemodynamically sound, complications in other systems have been controlled and preoperative medications have been reinstituted.

A typical problem is the patient who requires propranolol before operation, has surgery, and cannot take oral medications for a period of time after operation. Such a patient should have an intravenous propranolol regimen planned and instituted until oral medication can be resumed. Chung $^{25}$ has recommended that 10 per cent of the patient's daily oral dose be given intravenously over 24 hours.

The following brief case presentation illustrates the successful use of intravenous propranolol in the postoperative period. A 57 year old female with disseminated arteriosclerotic disease, moderately obese, hypertensive (170/100 torr), taking insulin for the control of diabetes, had a carotid endarterectomy and a myocardial infarction 18 months and 12 months respectively before her admission for a cholecystectorny. She had angina which was controlled by propranolol $180 \mathrm{mg}$ four times a day, and sublingual nitroglycerin for anginal attacks. Preoperatively her heart rate was $64 \mathrm{bpm}$ with an RPP of 11,000 , which did not produce signs or symptoms of myocardial ischaemia. The decision was made to give her intravenous propranolol $3 \mathrm{mg}$ per hour in the post-operative period to maintain her heart rate between 60 and $70 \mathrm{bpm}$. If the RPP rose over 11,000 , a vasodilator would be administered. This regime was to be continued until she was taking her propranolol orally, was haemodynamically sound, and considered safe to be discharged to ward care. Figure 7 illustrates 


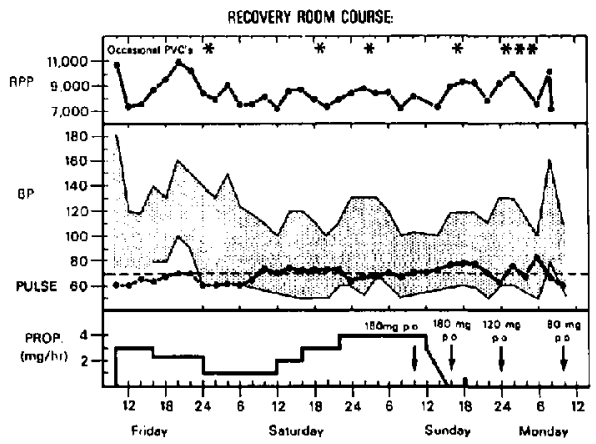

FIGURE 7 Rate pressure product, blood pressure, pulse rate, intravenous and oral propranolol regimen in a patient following cholecystectomy. Occasional PVC's occurred in spite of good control of the rate pressure product and fairly good control of the pulse rate.

her course in the recovery room and the following points can be made. She was started on propranolol $3 \mathrm{mg}$ per hour on arrival in the recovery room and this was maintained until early in the evening of the operative day, at which time she was doing well; in fact so well that the physician in charge of the recovery room reduced the propranolol to $1 \mathrm{mg}$ per hour. By mid-day of the second post-operative day her heart rate had begun to accelerate over the desired rate and the propranolol was gradually increased over a period of hours to a point where she was receiving $4 \mathrm{mg}$ per hour. This was maintined until she began to take her propranolol orally on the third post-operative day. Her RPP was always below 11,000 and, despite this and farily good control of her heart rate, she had occasional episodes of PVCs. At no time did she have angina and, in fact, her recovery room course was fairly uneventful. The procedure had been explained to the patient preoperatively and she actually felt extremely secure in the recovery room, requiring reassurance when she was being discharged from that ' rea. In retrospect, tighter control of the heart rate would probably have been achieved if the original plan to maintain her on propranolol $3 \mathrm{mg}$ per hour had been followed, and the PVCs might have been prevented. The only monitoring that this patient had was an ECG and an arterial line from which blood pressure was displayed continuously and blood gases sampled periodically. The decision had been made not to use a pulmonary artery catheter because she had been well controlled on propranolol before operation, the operation was to be a brief one, propranolol was to be reinstituted immediately following operation and, should any difficulties arise in the recovery room, a pulmonary artery catheter could easily be introduced. Had postoperative hypertension, ischaemic signs on the ECG or angina occurred, we were prepared to give her intravenous nitroglycerin. This case is an example of the minimal amount of intervention that one should be prepared to undertake in a patient with severe ischaemic heart disease if one is to have reinfarction rates which approach those reported by Rao. ${ }^{8}$

\section{SUMMARY}

It is difficult to pick out a sensitive marker for the effectiveness with which patients with heart disease have been managed for non-cardiac operations. Reinfarction rates have frequently been cited for this purpose over the past 20 years and it is interesting and perplexing that, until very recently, we have been unable to show significant decreases in reinfarction rates in patients who have had a myocardial infarction within six months of surgery. It has been our opinion for a number of years that, in general, patients with heart disease are well managed in the intraoperative phase of their hospital stay. The successful management of patients during this period is based on the fact that the majority of anaesthetists now appear to have a good understanding of the pathophysiology of heart disease and are becoming more aggressive in the monitoring and treatment of abnormal haemodynamics when they occur. The ultimate extent to which morbidity and mortality can be reduced in patients with heart disease for non-cardiac surgery will not be known until patients at high risk receive the same intensive postoperative care that patients who undergo heart surgery enjoy. It is the fact that patients are haemodynamically stable and do not have myocardial ischaemia throughout the peri-operative period that is important, rather than the way in which it is achieved.

\section{REFERENCES}

1. Wynands, J.E., Sheridan, C.A., Batra, M.S., Palmer, W.H. \& Shanks, J. Coronary artery disease. Anesthesiology 33: 260 (1970).

2. SLOGOFF, S. Anesthetic considerations for the patient with ischemic heart disease. ASA refresher courses in anesthesiology, 8: 179 (1980).

3. Wynands, J.E. The high risk cardiac patient undergoing general surgery. Can. J. Surg. 21: 475 (1978) 
4. Tarhan S., White, R.D. \& Moffitt, E.A. Anesthesia and postoperative care for cardiac operations. Ann. Thor. Surg. 23: 173 (1977).

5. TOPkINS, M.J. \& ARtusio, J.F. Myocardial infarction and surgery. A five year study. Anesth. Anal. 43: 716 (1964)

6. Tarhan, S., Moffitt, E.A., TAYlor, W.F. \& GIULIANI, E.R. Myocardial infarction after general anesthesia. J.A.M.A. 220: 1451 (1972).

7. Steen, P.A., Tinker, J.H. \& Tarhan, S. Myocardial reinfarction after anesthesia and surgery. J.A.M.A. 239: 2566 (1978).

8. RAO, T.L.K. \& EL-ETR, A.A. Myocardial reinfarction following anesthesia in patients with recent infarction. Anes. Anal. 60:271 (1981).

9. Lappas, D.G., Fahmy, N.R., Ohtaka, M., Moss, J. \& SLATER, E.E. Interaction of reninangiotensin-catecholamines in cardiac surgical patients. Anesthesiology 51: S98 (1979).

10. OKada, R.D., Pohost, G.M., Kitshenbaum, H.D., KuSHNER, F.G., BOUCHER, C.A., Block, P.C. \& Strauss, H.W. Radionuclidedetermined change in pulmonary blood volume with exercise. Improved sensitivity of multigated blood-pool scanning in detecting coronary-artery disease. New Engl. J. Med. 301: 569 (1979)

11. LAPPAS, D.G. Cardiac dysfunction and its treatment. ASA refresher courses in anesthesiology. 8: 87 (1980)

12. Goldman, L., Caldera, D.L., Nussbaum, S.R., Southwick, F.S., Krogstad, D., MuRRaY, B., Burke, D.S., O'Malley, T.A., Goroll, A.H., Caplan, C.H., Nolan, J., CaraBELLO, B. \& SLATER, E.E. Multifactorial index of cardiac risk in noncardiac surgical procedures. New Eng. J. Med. 297: 845 (1977).

13. MERin, R.G. The function of the heart: effects of anesthetics and adjuvant drugs. ASA refresher courses in anesthesiology. 6:81 (1978).

14. Kaplan, J.A. Monitoring of myocardial ischemia during anesthesia. ASA refresher courses in anesthesiology 7: 155 (1979)
15. RoY; W.L., Edelist, G. \& GILbert, B. Myocardial ischemia during non-cardiac surgical procedures in patients with coronary-artery discase. Anesthesiology 51:393 (1979).

16. BYRICK, R.J. \& NOBLE, W.H. Influence of elevated pulmonary vascular resistance on the relationship between central venous pressure and pulmonary artery occluded pressure following cardiopulmonary bypass. Can. Anaesth. Soc. J. 25: 106 (1978).

17. Cokkinos, D.V. \& Voridis, E.M. Constance of pressure-rate product in pacing-induced angina pectoris. Br. Heart J. 38:39 (1976)

18. Robinson, B.F. Relation of the heart rate and systolic blood pressure to the onset of pain in angina pectoris. Circulation 35: 1073 (1967)

19. LowENSTEIN, E. Anesthetic considerations in coronary-artery disease. ASA refresher courses in anesthesiology. 4:51 (1976).

20. Brown, B.R. JR. Anesthesia and essential hypertension. ASA refresher courses in anesthesiology. 7: 41 (1979).

21. Slogoff, S., KeAts, A.S. \& OTt, E. Preoperative propranolol therapy and aortocoronary bypass operation. J.A.M.A. 240: 1487 (1978),

22. ROIZEN, M.F., HAMILTON, W.K. \& SoHN, Y.J. Treatment of increased PCWP by increasing the dose of enflurane or halothane. Anesth. Anal. 60: 273 (1981).

23. Quintin, L., Whalley, D.G., Wynands, J.E., Morin, J.E. \& Burke, J. Oxygen high dose fentanyl anesthesia for aorto-coronary by. pass surgery, Can. Anaesth. Soc. J. 28: 314-320 (1981).

24. CULLEN, D.J. Recovery room care of the surgical patient. ASA refresher courses in anesthesiology. 8: 13 (1980).

25. CHuNG, D.C. Anaesthetic problems associated with the treatment of cardiovascular disease: II. Beta-adrenergic antagonists. Can. Anaesth. Soc. J. 28: 105 (1981) 